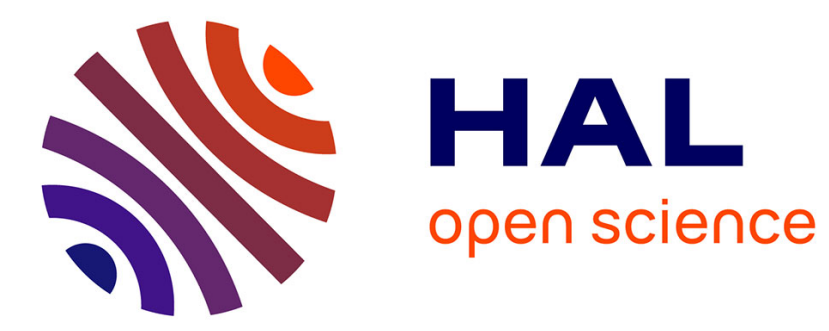

\title{
New metrics to evaluate mobility models properties
}

Fabrice Theoleyre, Rabih Tout, Fabrice Valois

\section{To cite this version:}

Fabrice Theoleyre, Rabih Tout, Fabrice Valois. New metrics to evaluate mobility models properties. International Symposium on Wireless Pervasive Computing. ISWPC, Feb 2007, San Juan, Puerto Rico. pp.1-6, 10.1109/ISWPC.2007.342624 . hal-00371390

\section{HAL Id: hal-00371390 https://hal.science/hal-00371390}

Submitted on 27 Mar 2009

HAL is a multi-disciplinary open access archive for the deposit and dissemination of scientific research documents, whether they are published or not. The documents may come from teaching and research institutions in France or abroad, or from public or private research centers.
L'archive ouverte pluridisciplinaire HAL, est destinée au dépôt et à la diffusion de documents scientifiques de niveau recherche, publiés ou non, émanant des établissements d'enseignement et de recherche français ou étrangers, des laboratoires publics ou privés. 


\title{
New metrics to evaluate mobility models properties
}

\author{
Fabrice Theoleyre, Rabih Tout, Fabrice Valois \\ CITI Laboratory - INRIA Rhône Alpes - INSA Lyon \\ 21, avenue Jean Capelle - 69621 Villeurbanne Cedex - France \\ Email: \{firstname.lastname\}@insa-lyon.fr
}

\begin{abstract}
Mobility models allow to mimic the behavior of mobile nodes when MANET performances are evaluated via simulations. Nevertheless, simulations results are strongly correlated with the mobility model. In this work, we introduce new metrics in order to characterize any mobility model. These metrics reflect the nodes distribution, the local topology changes, the repetitive behavior of a model,etc. They allow in consequence to characterize mobility models, and particularly to introduce a taxonomy based on objective and quantitative criteria. Using these metrics, we characterize some individual and one group mobility models. Keywords- mobility models, characterization, metrics, taxonomy
\end{abstract}

\section{INTRODUCTION}

Mobile Ad Hoc Networks (MANET) are literally networks ready to work. All mobile terminals can communicate with other nodes via wireless communications. The network must function autonomously, without any human intervention. Many protocols were proposed to deal with these networks [1] and their performances were mainly evaluated through simulations. The perfect way to mimic the nodes mobility is to inject real movements in the simulation. In this way, we can efficiently evaluate the performances of one protocol in a given application. However, MANET are currently rarely deployed, and no MANET traces are available. Thus, many analytical mobility models were proposed in order to simulate the displacement of a node or of a group of nodes. Since ad hoc networks are promised to a large spectrum of utilization, many different mobility models exist in the literature ([2] presents a detailed survey). Since these models are very different, they greatly impact the performance evaluation.

The main contribution of this work is the proposition of new metrics to discriminate and classify mobility models. We introduce metrics to evaluate the network dynamicity, the neighborhood persistence and the homogeneity of the network related to the mobility models by themselves. Consequently, we can propose an objective taxonomy of mobility models, based on quantitative metrics. These metrics can constitute a framework to determine the behavior of a new mobility model, and eventually to determine its redundancy with another mobility model. In the same way, from real MANET testbed and using these metrics we can identify the most suited analytical mobility model. It could be helpful to evaluate the performances through simulations. The reader can verify that these metrics are independent from any protocol. In particular, they do not integrate routing metrics since we want to characterize only mobility models which impact any upper layer, and not only the routing layer (services discovering, mobility management or the MAC layer for example).
This work is organized as follows. Related works are introduced in II. Section III presents the metrics to classify and discriminate mobility models. In particular, new metrics characterizing the network dynamic and distribution are detailed. A performance evaluation is presented in section IV. Finally, section $\mathrm{V}$ concludes this work nd gives some perspectives.

\section{RELATED WORK}

The simulations represent the most common tool to evaluate the performances of new protocols in MANET. Thus, many mobility models were proposed to mimic the behavior of independent mobile nodes. [2] gives an overview of these models. We will present here a short overview of the main mobility models, and will give a taxonomy to classify all these models. We will verify the relevance of this taxonomy to discriminate different mobility models in the performance evaluation, in section IV.

\section{A. Individual models}

MANET are spontaneous networks: a node can freely enter in the network, and leave it at any time. Individual mobility models consider that all the nodes are independently mobile: in other words, a rule could be applied independently to each node to model its displacement. We chose to differentiate memory less and smooth models.

1) Memory less models: In memory less models, the direction and speed chosen by a node at the time $t$ is completely independent from the previous values at the time $t-\Delta(t)$.

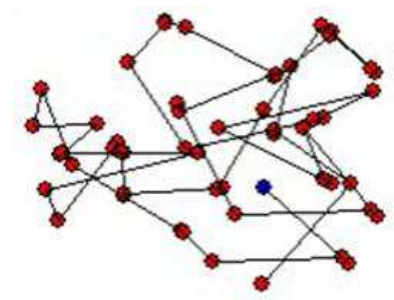

(a) random walk (time variant)

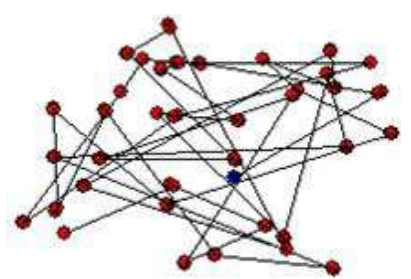

(b) random waypoint
Fig. 1. Mobility patterns of the random waypoint and random walk models

The random walk is a very simple model: a node chooses randomly a direction (between 0 and $2 \Pi$ ) and a speed (between $V_{\min }$ and $\left.V_{\max }\right)$. Then, it is moving during the duration $t$ or the distance $d$. Then, the node chooses random new values (fig. 1(a)). According to [3], this model was first introduced by Einstein in 1926. 
The random waypoint is the largest used model: a node chooses a random destination (in the simulation area) and speed (between $V_{\min }$ and $V_{\max }$ ). When the node reaches this destination, the algorithm reiterates after a pause. This mobility model was first introduced to evaluate the performances of DSR[4], a routing protocol for ad hoc networks (fig. 1(b)).

The random direction mobility model was proposed to overcome the problems of the random waypoint model, in order to distribute more uniformly the points in the simulation area. A node chooses a random speed (between $V_{\min }$ and $V_{\max }$ ) and direction (between 0 and $2 \Pi$ ) and changes these values only when it reaches the boundaries of the simulation area. Eventually, the node takes a pause time before moving (fig. 2(a)).

2) Smooth models: In the memory less models, the mobility pattern is very chaotic. Smooth models try to create a more realistic behavior. In the boundless mobility model [5], the position $(\mathrm{x}, \mathrm{y})$ and speed $\mathrm{v}$ at time $t+\Delta(t)$ are correlated with the speed and position at time $t$. More precisely, the speed (resp. direction) could change according to a small difference $\Delta(v) \cdot \Delta(t)$ (resp. $\Delta(\Theta) \cdot \Delta(t)$ ). The Gauss-Markov model [6] is similar to the boundless mobility model since position and speed are time-correlated. However, the variations respect a gaussian distribution.

The markovian random path [7] proposes to use a markov chain to model the mobility: one chain for the horizontal displacement, and another chain for the vertical displacement. A transition matrix allows to configure the probability to change the directions when another value must be chosen.

Finally, the city section model [8] allows to model the mobility of a node in a urban environment. The node will uniquely move along some particular horizontal and vertical paths. Eventually, a different speed could be specified for each street.

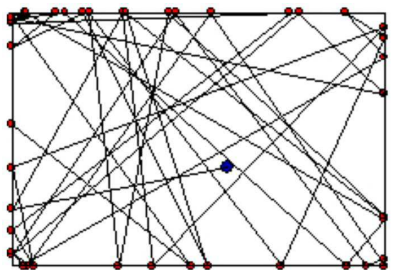

(a) random direction model

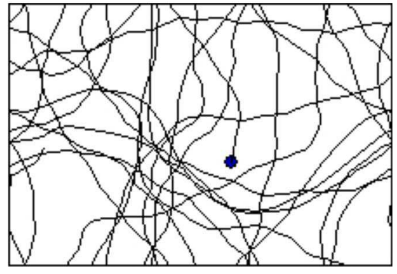

(b) boundless model
Fig. 2. Mobility pattern of the random direction and boundless models

\section{B. Group models}

Some articles try to model the mobility of groups of nodes. These models are widely based on the existence of reference points: one or several nodes are moving in a small area, surrounding a common reference point.

In the column model[3], several reference points are defined, forming a column. This column is moving linearly according to the same individual mobility model: all the reference points keep on constituting a column. A node corresponds to each reference point. Eventually, a node can moves around its reference point according to a different mobility model (but it must stay close to this reference) (fig. 3(a)). This model is widely used to model the displacements in a battlefield. The nomadic community model [9] follows a similar approach: each community is represented by a reference point (fig. 3(b)). The reference is moving according to a smooth individual mobility model. Then, all other nodes are moving according to the random waypoint model, in a small area surrounding this reference point. This could model well the displacement of a group of turists.

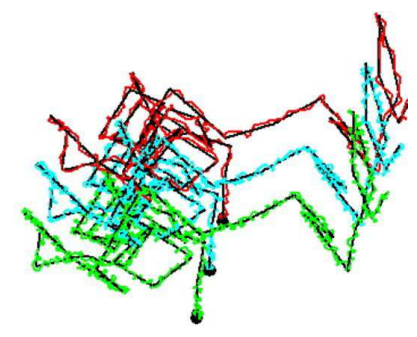

(a) column model

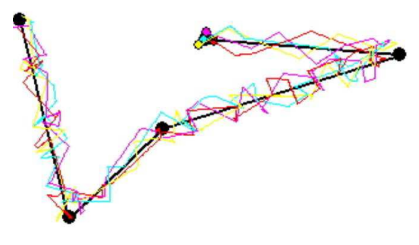

(b) nomadic community model
Fig. 3. Mobility pattern of the different group mobility models

\section{Properties}

Several analysis were conducted to study the behavior of different mobility models. Since the random waypoint model is the most used model, it focused the efforts. [10] proves that the average speed decreases constantly, mainly because the node can choose a null speed, and thus never reaches the destination, blocked in a static state. [11] presents a method to accelerate the convergence of the random waypoint mobility model in re-injecting stationary values. [12] presents a stochastic study, proving in particular that the nodes are mainly present in the center of the simulation area.

\section{Metrics to ChARACTERIZE MOBility MODELS}

Many mobility models were already and keep on being proposed. Our goal is here to focus on how to discriminate different mobility models. These quantitative and objective metrics will allow to discriminate mobility models and eventually to verify the relevance and originality of a newly proposed model. Routing is a main application in MANET. Consequently, [13] chose to focus on routing efficiency metrics like the delivery ratio. However, these metrics are not directly related to the mobility model and should be, according to us, improved.

\section{A. Existing metrics}

We distinguish two types of existing metrics: the metric computed for each node according only to its own properties, and the metrics related to the interaction between different nodes.

1) Individual metrics: We can model the ad hoc network with a graph: a vertex corresponds to each node and there exists an edge between two vertices if the corresponding nodes can communicate with each other. Usually, we use the notation $\mathrm{G}(\mathrm{V}, \mathrm{E}): \mathrm{G}$ is the graph with the set of vertices $V$ and the set of 
edges $E$. If we consider only bidirectional communications, we can measure the degree of each node, i.e. the number of edges: it represents the number of radio neighbors (like [14]), impacting on the overhead for example. If the graph is asymmetrical, we can distinguish the inner and outer degree.

Besides, we can directly extract values like the direction, the absolute speed and the position of each node.

2) Complex metrics: More complex metrics are useful to extract the common behavior of the network. [15] measures the link duration, i.e. the average time before a radio link is broken. Eventually, thanks to this metric, we can compute the average duration of a path in the network (the probability of a path break being the product of each individual probability). If the ad hoc network is used for routing, this metric can reflect directly the reliability of a given route.

[16] proposes to estimate the correlation of the speed of a node for different time $t$ and $t+\Delta(t)$, called the degree of temporal dependence. This reflects the smoothness of a mobility model. The degree of temporal dependence is the product of the cosine formed by the angle of both directions with the ratio of both absolute speeds. More formally, let $\vec{v}(a)$ and $\vec{v}(b)$ the speed vectors of the same nodes at different instants (without lake of genericity, let $\|\vec{v}(a)\|<\|\vec{v}(b)\|)$. The degree of temporal dependence is:

$$
\frac{\vec{v}(a) \cdot \vec{v}(b)}{\|\vec{v}(a)\| \cdot\|\vec{v}(b)\|} \cdot \frac{\| \vec{v}(t+\Delta(a) \|}{\|\vec{v}(b)\|}
$$

This formula is also useful to describe the correlation between the speed of two different nodes, metric called the degree of spatial dependence.

\section{B. New metrics}

However, these metrics are not reflecting well all the mobility models properties. In particular, the network dynamicity is not completely represented. Indeed, [12] demonstrates for example the non-uniform distribution of the nodes in the simulation area with the random waypoint mobility model. However, none of these metrics could express such a property. Consequently, we introduce here new metrics to fully characterize the behavior of a mobility model.

1) network diameter: If we model a MANET with a graph, we can measure the graph diameter, i.e. the maximum distance in hops between two nodes. More formally, let $\lg (a, b)$ be the minimum number of edges required to find a connected path between $a$ and $b$. Then, diameter $=\max \{\lg (a, b)\}_{\forall(a, b) \in V^{2}}$

The network diameter is representative of the average length of the paths of the network. Thus, a mobility model which creates a low diameter will for example improve routing performances, minimizing interferences. In particular, we can verify that the network diameter represents a stable value, not changing importantly when the nodes are moving.

The reader can remark that, although this metric can reflect routing performances, it is related only to the mobility model by itself, independent from any particular protocol.
2) neighborhood instability: The stability of the neighborhood impacts several protocols. For example, when a node offers a service to its neighbors, an important neighborhood instability will decrease the reliability. We estimate the neighborhood instability as the number of radio link apparition and disappearance. It is normalized by the total number of radio links.

It is important to monitor the distribution of the neighborhood instability: with an high probability, a few nodes, very mobile, will know an important instability whereas other nodes, more statical will have an acceptable instability. In particular, a smooth model will tend to limit the neighborhood instability. On the contrary, a memory less will introduce high variations.

3) nodes distribution: We propose to measure the geographical nodes distribution. We represent graphically the node density in some small surface areas. This allows to have a snapshot of the nodes distribution. In particular, we can verify that the distribution is homogeneous, and not concentrated around the center of the simulation area, like with the random waypoint model.

More precisely, we cut the network in a regular grid of side $c$. Then, we count the number of nodes lying in each case. This number gives a color level to represent graphically the density in this particular cell.

This tool will give us the opportunity to verify that a mobility model does not present side effects. This also allows to differentiate heterogeneous and homogeneous models, i.e. models were the nodes are concentrated on restricted areas.

4) repetitive behavior: A realistic movement pattern is often repetitive. For example, a person will go working during the morning and come back home after his work. It could be interesting to extract a quantitative metric, related to the property of a node to exhibit the same movement after a given time.

To evaluate the repetitive behavior of a mobility model, we measure the average ratio of time during which a node is located inside the transmission range area of its initial position (cf. fig. 4). An important value of the repetitive metric means that the mobility model exhibits an important repetitive behavior. It could be the case if the direction and speed remain unchanged, or if the node is mobile only around a short distance from the initial position.

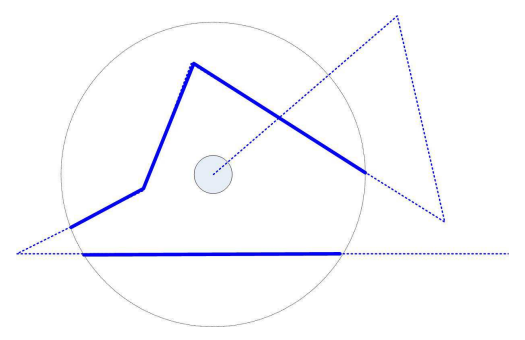

Fig. 4. Repetitive metric

5) clustering coefficient: The clustering coefficient was firstly introduced by Watts \& Strogatz[17] to measure the small-world graph property of a node. We propose to re-use 
this metric to discriminate mobility models. The clustering coefficient is the ratio of the radio links among neighbors and the number of neighbors.

More formally, let $N(u)$ be the set of neighbors of the node u. The clustering coefficient is defined as:

$$
\frac{\sum_{v \in N(u)}|\{x \in N(v)\}|}{N(u)}
$$

This clustering coefficient is related to the network redundancy. Indeed, when a node presents an high clustering coefficient, this means that its neighbors have many radio links with each other. Thus, this node is redundant: a route can, with an high probability, pass through its neighbors without a path length increase.

\section{MOBILITY MODELS CHARACTERIZATION}

In this section, we present a set of simulation results allowing to discriminate and classify different mobility models.

\section{A. Methodology}

We use the OPNET Modeler tool in order to simulate the MANET. We implemented six individual and one group mobility models: the random way point, random walk, random direction, boundless, Gauss-Markov, restricted random way point and the nomadic community (cf. [2]). We measured the metrics (in particular the five new metrics described above) presented in the section III. We run a set of at least 10 simulations for each result presented here. Moreover, the $95 \%$ confidence interval is systematically reported.

\section{B. Results}

We mainly investigated the neighborhood behavior (chaotic or stable), the link duration, the repetitive behaviors of the mobility models, and the nodes distribution in the simulation area. The results are deeply explained, giving ideas to introduce an objective taxonomy. By default, we assume a simulation area of $1000 * 1000 \mathrm{~m}$ and a simulation time of 1000 seconds. The radio range is assumed to be equal to $100 \mathrm{~m}$.

1) Neighborhood dynamic: The performances of mobile ad hoc networks are linked to the persistence of the neighborhood. For instance, the more the neighborhood of a node is stable, the more routing protocols are efficient: the network is more stable and the routes break less often. Figure 5 illustrates the impact of the network cardinality on the neighborhood stability. There are 3 kinds of mobility models: $i$ ) the smoothest models are the random direction and a group model (nomadic community). For a group model, it is clear that the neighborhood is stable because the main neighorhood of a node is restricted to the group. For the random direction, this result may appear more surprising but it is due to the bound effect: when a bound is reached, a node waits a time and then, changes of direction and go to another bound. If the pause time is important, the nodes have an high probability to be on the simulation area boundaries, stabilizing the topology. ii) The second part of mobility models exhibit a chaotic behavior: the neighborhood is not stable. This is the case for the random walk, the
Gauss-Markov, etc. iii) Finally, the random way point and the restricted way point exhibit a compromise behavior. In conclusion, if we fix the parameters of a given routing protocol, ir performances will be, with an high probability, better with the random waypoint than with the random walk mobility model. And the results may be better if a random direction is used. Clearly, the results of a performance evaluation of a routing protocol are greatly impacted by the mobility model.

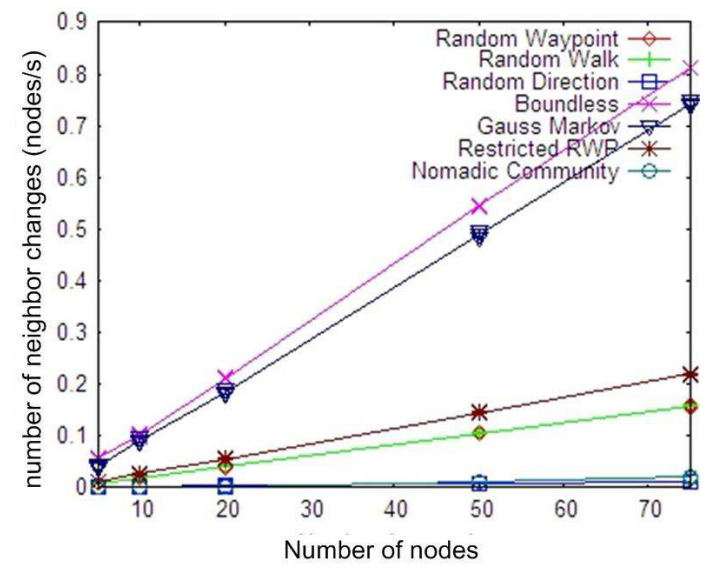

Fig. 5. Neighborhood stability: influence of network cardinality

Figure 6 investigates also the neighborhood stability but if the average node speed increases for a topology of 40 nodes. The impact of the speed on the neighborhood stability seems limited, except for the boundless and the Gauss-Markov mobility models.

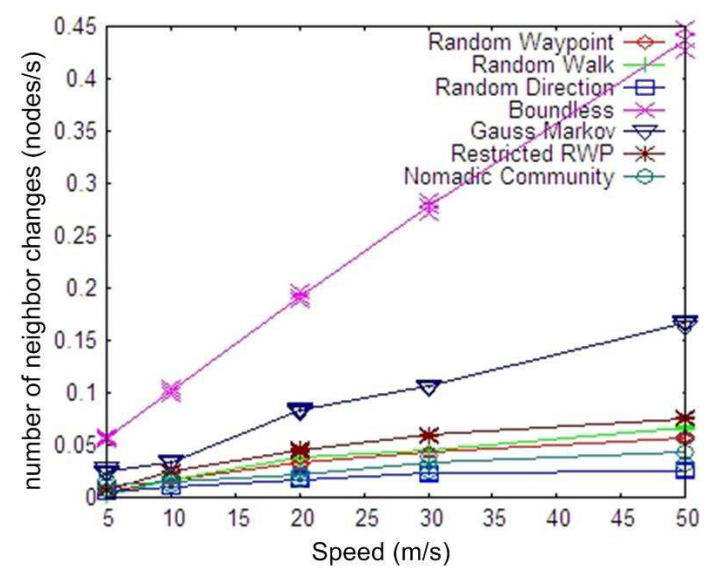

Fig. 6. Neighborhood stability: influence of node's speed

2) Link duration: After the node stability, we focus on the radio links changes. The link duration metric represents the average time of existence of a radio link between two nodes.

It is more a miscroscopic point of view. In fact, as it is shown on figure 7 the link duration is not impacted by the network cardinality for all the mobility models. Indeed, when the number of nodes increases, more radio links appear, increasing the absolute changes in the neighborhood. However, 
the mean duration of a particular radio links remains unchanged since the displacement does not change either.

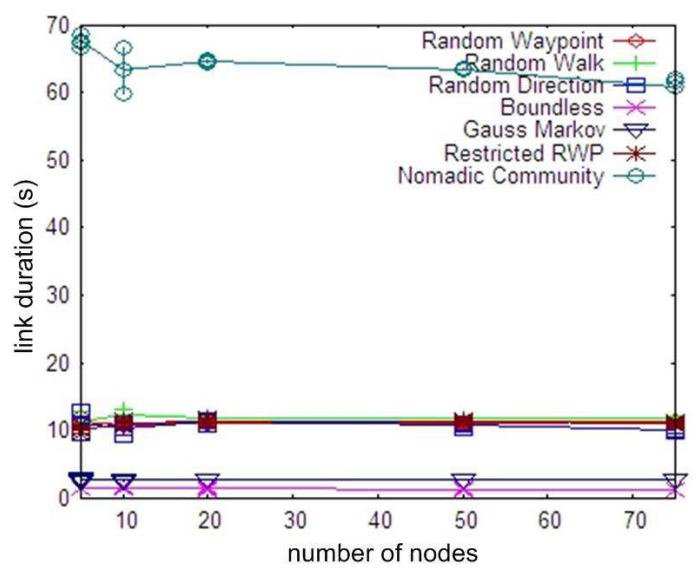

Fig. 7. Link duration: influence of network cardinality

But the link duration decreases drastically when the speed increases (figure 8). Indeed, when the speed increases, two nodes which are not moving in the same direction will quickly break their common radio link. Consequently, the link duration decreases drastically when the absolute speed increases. For example, this means that a services discovering protocol must re-trigger a new discovering very oftenly when the speed increases.

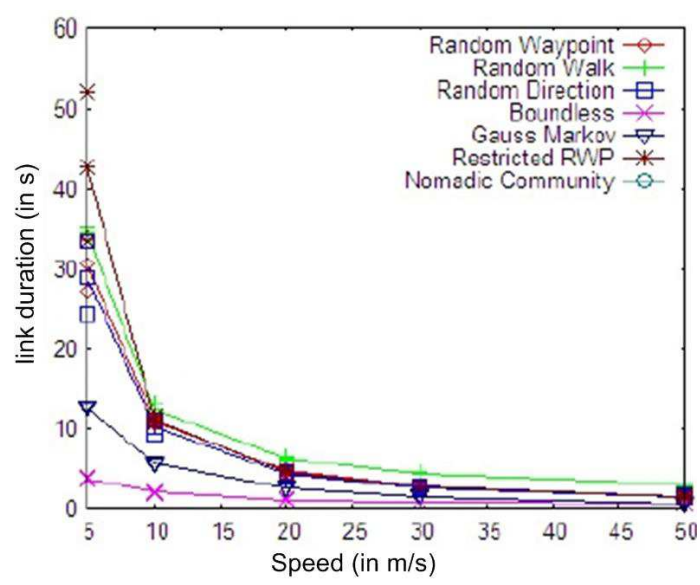

Fig. 8. Link duration: influence of node's speed

3) Repetitive behavior: In the repetitive behavior metric, we want to investigate if the mobility models lead to a repetitive behavior of node (figure 9). Thanks to the repetitive metric, we want to explore the reproctibility property of a given mobility model: does a node has an high probability to move according to a repetitive displacement pattern. It means, considering the initial position of a node and a service area around this initial position, during the simulation, what is the ratio of time during which a node stays around this initial service area? The boundless appears the less repetitive: this result will be confirmed by the next parameter (the probability of density is uniform with this mobility model, as shown below). The most repetitive model is the random walk model: a node has an high probability to stay around its initial position, limiting the displacements. The nomadic community model is quite an exception: a group of nodes will stay around the leader. The others mobility models present a very close repetitive property.

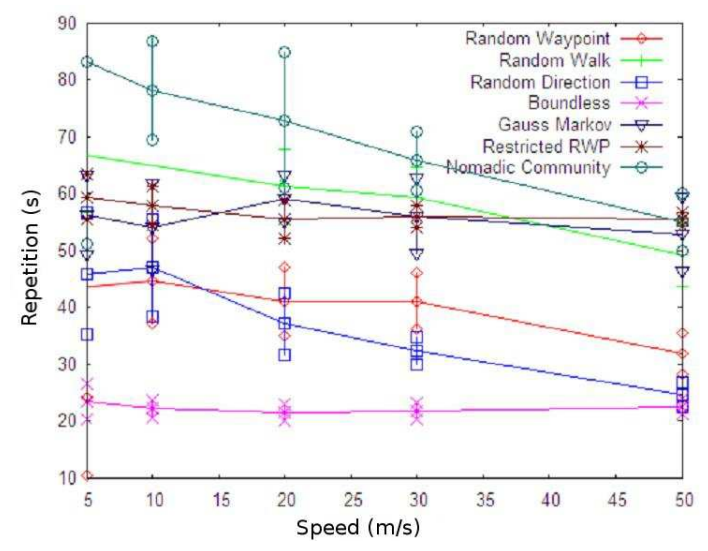

Fig. 9. Repetition: impact of the mobility

4) Nodes distribution: Finally, we studied the distribution of a nodes in the simulation area. In particular, we verified that the mobility models do not present side effects. The figures (10(a), 10(b), 11(a), 11(b)) illustrate this property. When a case of the grid is red, this means that the probability of density for this particular case is important.

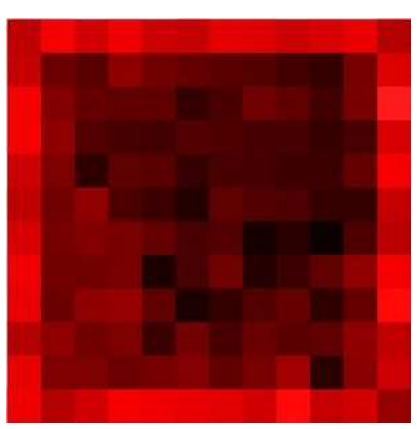

(a) Gauss-Markov

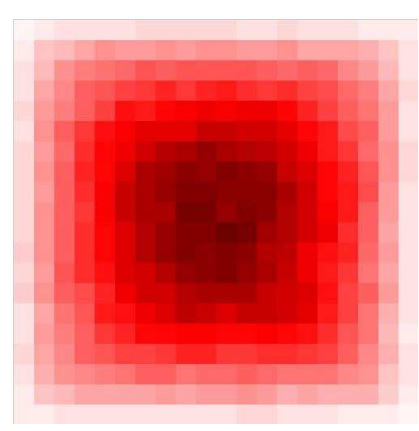

(b) Random Way Point
Fig. 10. Gauss-Markov and Random Way Point nodes distribution

The Markov mobility model (and also the Boundless, not represented here due to the lack of place) is the most uniform mobility model (fig. 10(a)): a node has no privileged location in the simulation area. On the contrary, the random waypoint (fig. 10(b)) exhibits a centralized behavior in the center of the simulation area: clearly, this will lead to important density in the center of the simulation area, creating an heterogeneous network.

Figures 11(a) and 11(b) illustrate the nodes distribution in the case of the random direction mobility model and the random walk, respectively. These two models appear homogenous in terms of nodes distribution over the simulation area. The simulation area boundaries represent a particular case for the 
random direction mobility model: a node must implement a pause when it reaches the simulation borders. However, if the pause time is null, the mobility model presents an uniform distribution.

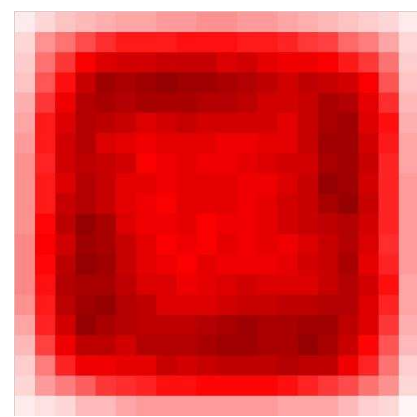

(a) Random Walk

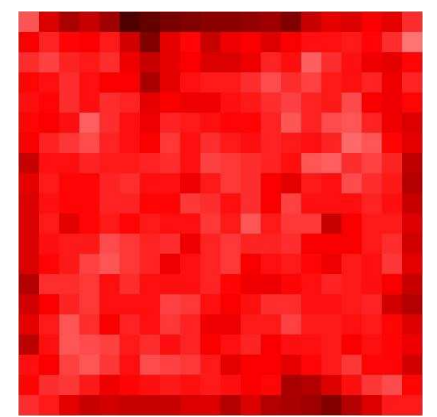

(b) Random Direction
Fig. 11. Random Walk and Random Direction nodes distribution

\section{Conclusion \& Perspectives}

MANET protocols are often evaluated through simulations, requiring mobility models which mimic the real movements of each node. Since MANET are promised to a wide spectrum of utilizations, many mobility models were proposed in the literature, presenting different behaviors. In particular, the performance evaluation of a MANET protocol will be widely impacted by the model. We proposed here five new metrics to discriminate them, introducing an objective and quantitative taxonomy. This characterization allows to understand deeply the behavior of some mobility models. Moreover, this framework allows to extract the most relevant mobility model for a particular application. In this way, the performances evaluation via simulations will be the most relevant as possible.

As a future work, we want to explore some new additional metrics to characterize dynamical graph properties to complete this framework. Moreover, we would like to validate this approach: we plan to set up an experimental MANET in order to collect statistics about the displacement of users. Then, we should obtain the mobility model most suitable to mimic this application, thanks to the metrics presented in this article. Finally, it could be relevant to propose new mobility models when no mobility model presents some expected characteristics (for example an important link duration with an high absolute mobility and an important clustering density).

\section{REFERENCES}

[1] I. Chlamtac, M. Conti, and J.-N. Liu, "Mobile ad hoc networking: imperatives and challenges," Ad hoc networks, vol. 1, no. 1, pp. 13-64, 2003.

[2] T. Camp, J. Boleng, and V. Davies, "A survey of mobility models for ad hoc networks research," Wiley Wireless Communications and Mobile Computing, vol. 2, no. 2, pp. 483-502, 2002.

[3] M. Sanchez and P. Manzoni, "Anejos: A java based simulator for adhoc networks.” Future Generation Computer Systems, vol. 17, no. 5, pp. 573-583, 2001.

[4] D. B. Johnson, D. A. Maltz, and Y.-C. Hu, "The dynamic source routing protocol for mobile ad hoc networks (DSR)," draft-ietf-manet-dsr-10.txt, IETF, Internet draft version 10, July 2004.
[5] Z. Haas, "A new routing protocol for reconfigurable wireless networks," in International Conference on Universal Personal Communications (ICUPC). San Diego, USA: IEEE, October 1997, p. 562565.

[6] B. Liang and Z. Haas, "Predictive distance-based mobility management for pcs networks," in INFOCOM, 1999.

[7] C. Chiang, "Wireless networks multicasting," Ph.D. dissertation, Department of Computer Science, University of California, 1998.

[8] V. Davies, "Evaluating mobility models within an ad hoc network," Master's thesis, Colorado School of Mines, 2000.

[9] M. Sanchez, "http://www.disca.upv.es/misan/mobmodel.htm."

[10] J. Yoon, M. Liu, and B. Noble, "Random waypoint considered harmful," in INFOCOM. IEEE, April 2003.

[11] W. Navidi, T. Camp, and N. Bauer, "Improving the accuracy of random waypoint simulations through steady-state initialization," The Colorado School of Mines, Tech. Rep. MCS-03-08, June 2003.

[12] C. Bettstetter, H. Hartenstein, and X. Perez-Cosat, "Properties of the random waypoint mobility model: epoch length, direction distribution, and cell change rate," in Symposium on Modeling, Analysis and Simulation of Wireless and Mobile Systems (MSWiM). ACM, Sept. 2002.

[13] J. Ng and Y. Zhang, "Impact of group mobility on ad hoc networks routing protocols," in International Conference on Advanced Communication Technology (ICACT). IEEE, February 2006.

[14] H. Uehara, J. Tsumochi, and K. Masayama, "Impact of mobility metrics on routing protocols for mobile ad hoc networks," in PACRIM, 2003.

[15] M. Gerharz, C. de Waal, M. Frank, and P. Martini, "Link stability in mobile wireless ad hoc networks," in Conference on Local Computer Networks (LCN). IEEE, Nov. 2002.

[16] F. Bai, N. Sadagopan, and A. Helmy, "IMPORTANT: a framework to systematically analyze the impact of mobility on performance of routing protocols for adhoc networks," in INFOCOM. IEEE, April 2003.

[17] D. Watts and S. H. Strogatz, "Collective dynamics of smallworld networks," Nature, 393: 440-442, 1998. 